\title{
Methods in phycology: using the best methods
}

\author{
Michael A. Borowitzka ${ }^{1,2}$ \\ Published online: 29 September 2020 \\ (C) Springer Nature B.V. 2020
}

A critical component of research is the use of correct methods. However, it has been my experience as an Editor that many studies either use either outdated methods where more accurate ones are available or inappropriate methods are used. The former leads to unreliable results and the latter leads to incorrect results. There appear to be two main reasons for this: (1) methods are selected by researchers based on those used by others without checking whether alternative and updated methods are available and/or (2) methods are used uncritically without understanding the principles underlying the method resulting in them being inappropriately applied. Unfortunately, it is often assumed that, if it is published, it must be correct. Furthermore, the use of different methods also makes it much harder to compare results from different studies. Some examples of particular areas where poor methodology often is used include the extraction and quantification of chlorophylls and carotenoids, and the use of molecular methods to identify algal strains. Surprisingly, even very fundamental methods such as the determination of growth rates and the calculation of these rates in microalgae are sometimes done incorrectly.

Although there are some compilations of standardized methods available (e.g., Stein 1973; Lobban et al. 1988; Andersen 2005; Moheimani et al. 2013), these are not consulted as often as they should be. However, some of these contain now outdated methods and they only cover some of the methods. Furthermore, a few detailed studied which have compared methods (and laboratories) have shown that in some cases results can vary significantly for a number of reasons which are as yet not fully understood (Laurens et al. 2012, 2014).

With the aim of providing researchers with an easily accessible and up-to-date guidance to the most up-to-date basic and advanced methods in phycology, the Journal of Applied
Phycology will be publishing short articles under the rubric "Phycological Methods." These articles are intended to provide a guide to specific methods, including the history of their development, the basic principles underlying the method, any potential issues which need to be considered, guidelines on any limitations and a description of the recommended method.

The first two articles in this new category are published in this issue.

\section{References}

Andersen RA (ed) (2005) Algal Culturing Techniques. Elsevier, Amsterdam

Laurens LML, Dempster TA, Jones HDT, Wolfrum EJ, Van Wychen S, McAllister JSP, Rencenberger M, Parchert KJ, Gloe LM (2012) Algal biomass constituent analysis: method uncertainties and investigation of the underlying measuring chemistries. Anal Chem 84: 1879-1887

Laurens LML, Van Wychen S, McAllister JP, Arrowsmith S, Dempster TA, McGowen J, Pienkos PT (2014) Strain, biochemistry, and cultivation-dependent measurement variability of algal biomass composition. Anal Biochem 452:86-95

Lobban CS, Chapman DJ, Kremer BP (eds) (1988) Experimental phycology. A Laboratory manual. Cambridge University Press, Cambridge

Moheimani NR, Borowitzka MA, Isdepsky A, Fon Sing S (2013) Standard methods for measuring growth of algae and their composition. In: Borowitzka MA, Moheimani NR (eds) Algae for biofuels and energy. Springer, Dordrecht, pp 265-284

Stein JR (ed) (1973) Handbook of Phycological methods. Culture methods and growth measurements. Cambridge University Press, Cambridge

Publisher's note Springer Nature remains neutral with regard to jurisdictional claims in published maps and institutional affiliations.

Michael A. Borowitzka

M.Borowitzka@murdoch.edu.au

1 Algae R\&D Centre, Murdoch University, Murdoch, WA 6150,

Australia

2 OzAlgae, P.O. Box 41, Cygnet, TAS 7112, Australia 